\title{
Proceeding
}

8th INSHS International Christmas Sport Scientific Conference, 5-7 December 2013. International Network of Sport and

Health Science. Szombathely, Hungary

\section{Technical movements in archery}

\author{
Daniela Tursi , Salvatore Napolitano \\ Department Physical Education and Wellness, University of Naples "Parthenope", Naples, Italy
}

\begin{abstract}
Tursi, D. \& Napolitano, S. (2014). Technical movements in archery. J. Hum. Sport Exerc., 9(Proc1), pp.S570S575. The archery is a typically closed skill sport. It is a "closed skill" sport characterized by repetition, as precise as possible, of a movement already known automated. The aim is to evaluate the effects of motor imagery practice in training. The motor imagery is a cognitive process of mental simulation of actions in absence of movement. There are two methods to improve skills learning through motor imagery: in first person and In third person. The biological basis on which the motor imagery theory is founded, is formed by: mirror neurons. It is an experimental approach and it consists of two steps. Both groups were video taped in the execution of sport skills and evaluated, through a check list made by archery indicators: positioning phase, traction and aim, release and follow through in 4 level of descriptors by technicians every four months. A sample of archery team of children (9-11 years old) splitting in control and experimental groups, after a pre-assessment, performed by technicians, about technical skills through an evaluation grid prepared "ad hoc" on technical fundamentals taken into the study to form two homogeneous groups (Experimental group $n=10$, control group $n=10$ ). Experimental group using modeling video performed by a athletes of National Italian Archery Team could lead to significant changes in technical skills. Data recruit at starting, ongoing and final training program and their analysis, evaluation and comparison by control group show percentage of $9 \%$ better outcomes. particularly, at final training shows a minimum improvement $3.2 \%$ than ongoing phase that gives a maximum improvement $9.6 \%$, while a substantial balance in the control group with a medium improvement of $3.2 \%$ in every phase of training program. In this study two basic aspects of the performance are examined: the motor execution and the motor imagine. Both share the same neuromotor mechanism: the motor imagery. Concerning the woman artistic gymnastics, it can be useful during the training and the race. So providing the athletes and trainers of a means which uses the motor imagery as a possible application for the improvement of the performance. So in conclusion, the study aims to provide a standard training feasible on a large scale to train the cognitive and physical abilities of an athlete and provide a support tool in the race in order to improve performance, optimize time and to reduce the margin of error. Keywords: MOTOR IMAGERY, ARCHERY, PERFORMANCES.
\end{abstract}

\footnotetext{
Corresponding author. Department Physical Education and Wellness, University of Naples "Parthenope", Naples, Italy. E-mail: daniela.tursi1@gmail.com 8th INSHS International Christmas Sport Scientific Conference, 5-7 December 2013. International Network of Sport and Health Science. Szombathely, Hungary. JOURNAL OF HUMAN SPORT \& EXERCISE ISSN 1988-5202 (c) Faculty of Education. University of Alicante doi:10.14198/jhse.2014.9.Proc1.48
} 


\section{INTRODUCTION}

The archery is a typically closed skill sport. It is a "closed skill" sport characterized by repetition, as precise as possible, of a movement already known automated. The aim is to evaluate the effects of motor imagery practice in training. The motor imagery is a cognitive process of mental simulation of actions in absence of movement. There are two methods to improve skills learning through motor imagery: in first person and in third person. The biological basis, on which the motor imagery theory is founded, is formed by: mirror neurons.

\section{METHODS}

It is an experimental approach and it consists of two steps. Both groups were video taped in the execution of sport skills and evaluated, through a check list made by archery indicators: positioning phase, traction and aim, release and follow through in 4 level of descriptors by technicians every four months. A sample of archery team of children (9-11 years old) splitting in control and experimental groups, after a pre-assessment, performed by technicians, about technical skills through an evaluation grid prepared "ad hoc" on technical fundamentals taken into the study to form two homogeneous groups (Experimental group $n=10$, control group $n=10$ ). Experimental group using modeling video performed by athletes of National Italian Archery Team could lead to significant changes in technical skills.

Table 1. Structure of the experimental group

\begin{tabular}{ccccc}
\hline gruppo sperimentale & scarso/ 0-3 & medio/4-5 & buono/6-7 & ottimo/8-10 \\
\hline $\begin{array}{c}\text { fase di posizionamento } \\
\text { trazione e mira }\end{array}$ & 6 & 3 & 1 & 0 \\
$\begin{array}{c}\text { release and follow } \\
\text { through }\end{array}$ & 5 & 4 & 1 & 0 \\
\hline
\end{tabular}

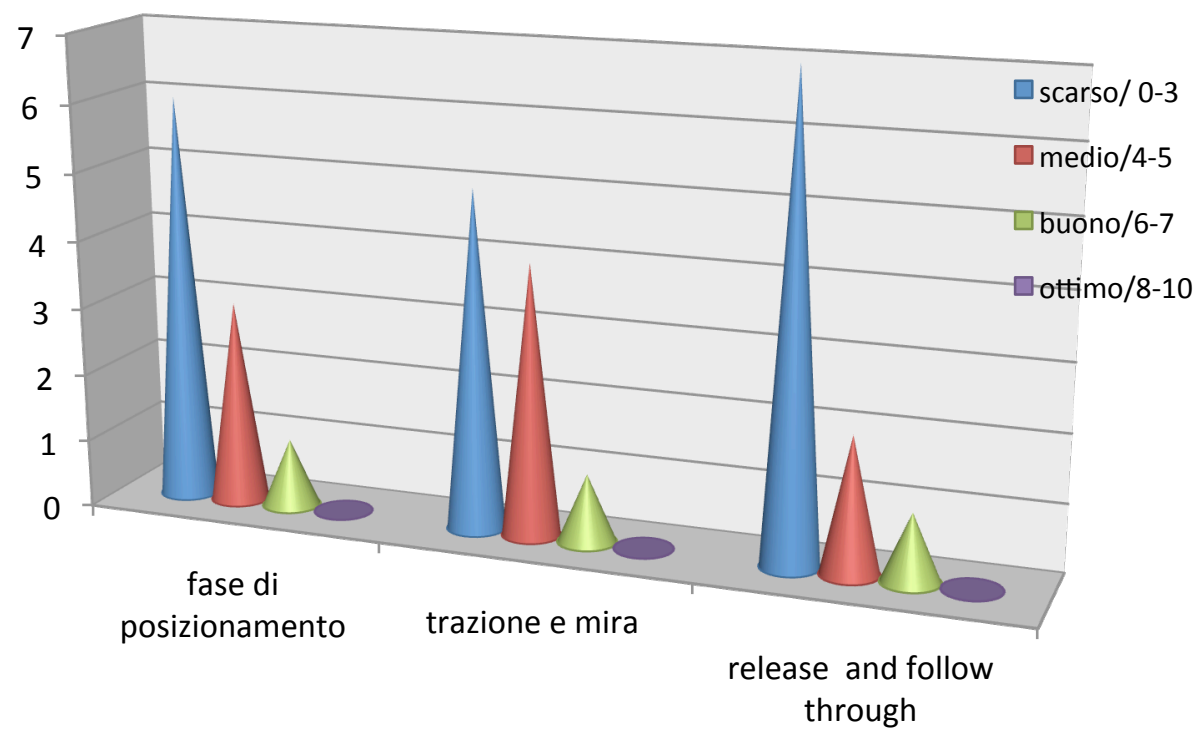

Figure 1. Initial phase - Experimental group 
Table 2. Structure of the control group

\begin{tabular}{ccccc}
\hline gruppo di controllo & scarso/ 0-3 & medio/4-5 & buono/6-7 & ottimo/8-10 \\
\hline $\begin{array}{c}\text { fase di posizionamento } \\
\text { trazione e mira }\end{array}$ & 7 & 2 & 1 & 0 \\
release and follow through & 6 & 3 & 1 & 0 \\
\hline
\end{tabular}

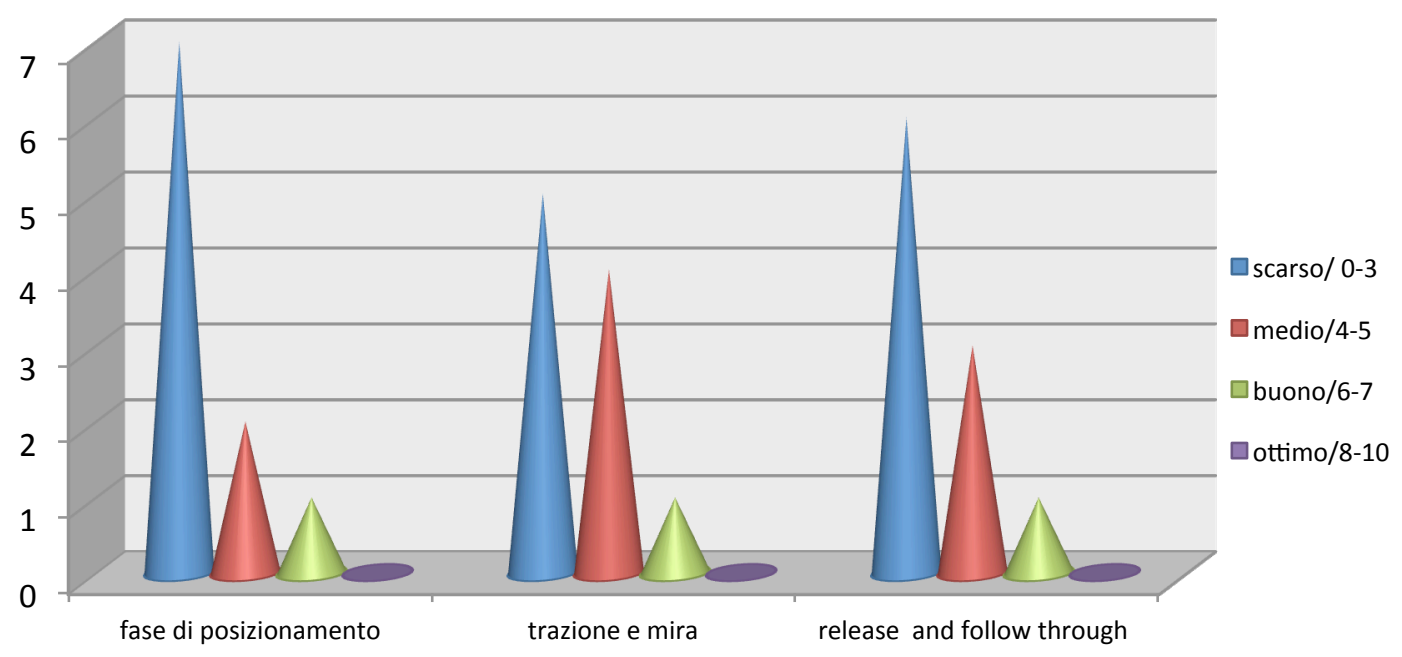

Figure 2. Initial phase - Control group

\section{RESULTS}

Table 3. Technical structure of the experimental group

\begin{tabular}{ccccc}
\hline Experimental gruop & scarso/ 0-3 & medio/4-5 & buono/6-7 & ottimo/8-10 \\
\hline fase di posizionamento & 3 & 4 & 2 & 1 \\
trazione e mira & 2 & 2 & 5 & 1 \\
release and follow through & 4 & 1 & 4 & 1 \\
\hline
\end{tabular}

Data recruit at starting, ongoing and final training program and their analysis, evaluation and comparison by control group show percentage of $9 \%$ better outcomes. particularly, at final training shows a minimum improvement $3.2 \%$ than ongoing phase that gives a maximum improvement $9.6 \%$, while a substantial balance in the control group with a medium improvement of $3.2 \%$ in every phase of training program. 


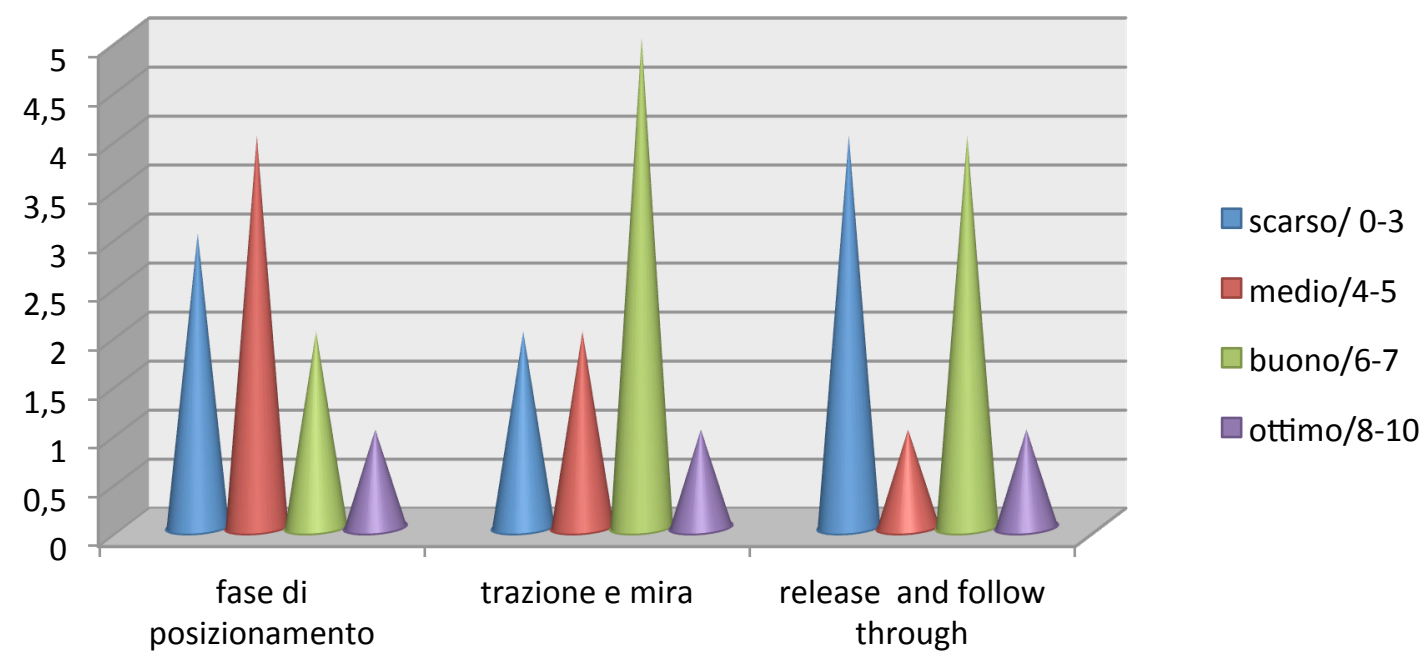

Figure 3. Final phase - Experimental Group

Table 4. Final phase - whole Group

\begin{tabular}{ccccc}
\hline gruppo campione & scarso/ 0-3 & medio/4-5 & buono/6-7 & ottimo/8-10 \\
\hline fase di posizionamento & 4 & 4 & 2 & 0 \\
$\begin{array}{c}\text { trazione e mira } \\
\text { release and follow } \\
\text { through }\end{array}$ & 4 & 4 & 2 & 0 \\
\hline
\end{tabular}

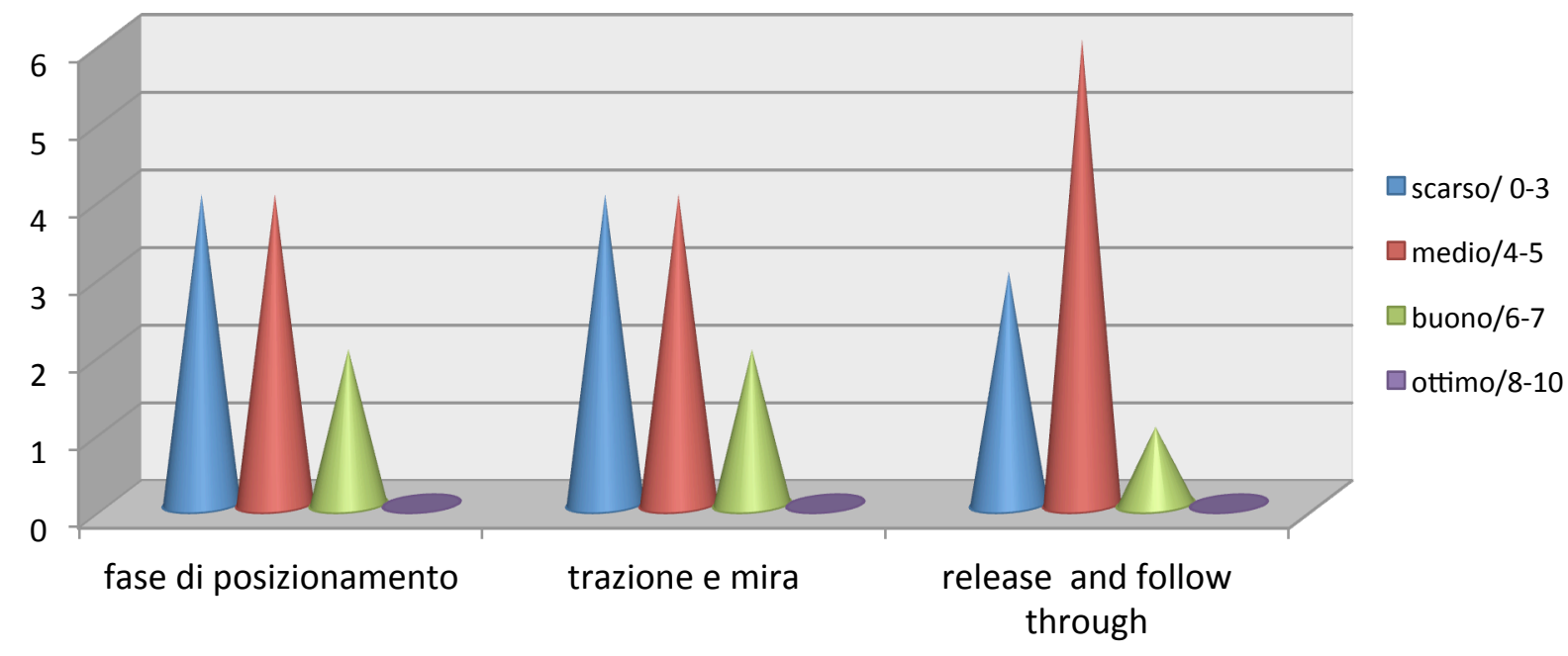

Figure 4. Final Phase - Control Group 
Table 5. Final scores

\begin{tabular}{ccccc}
\hline & scarso/ 0-3 & medio/4-5 & buono/6-7 & ottimo/8-10 \\
\hline fase di posizionamento g. e. & 3 & 4 & 2 & 1 \\
fase di posizionamento g. c. & 4 & 4 & 2 & 0 \\
trazione e mira g.e. & 2 & 2 & 5 & 1 \\
trazione e mira g. c. & 4 & 4 & 2 & 0 \\
release and follow through g.e. & 4 & 1 & 4 & 1 \\
release and follow through g.c. & 3 & 6 & 1 & 0 \\
\hline
\end{tabular}
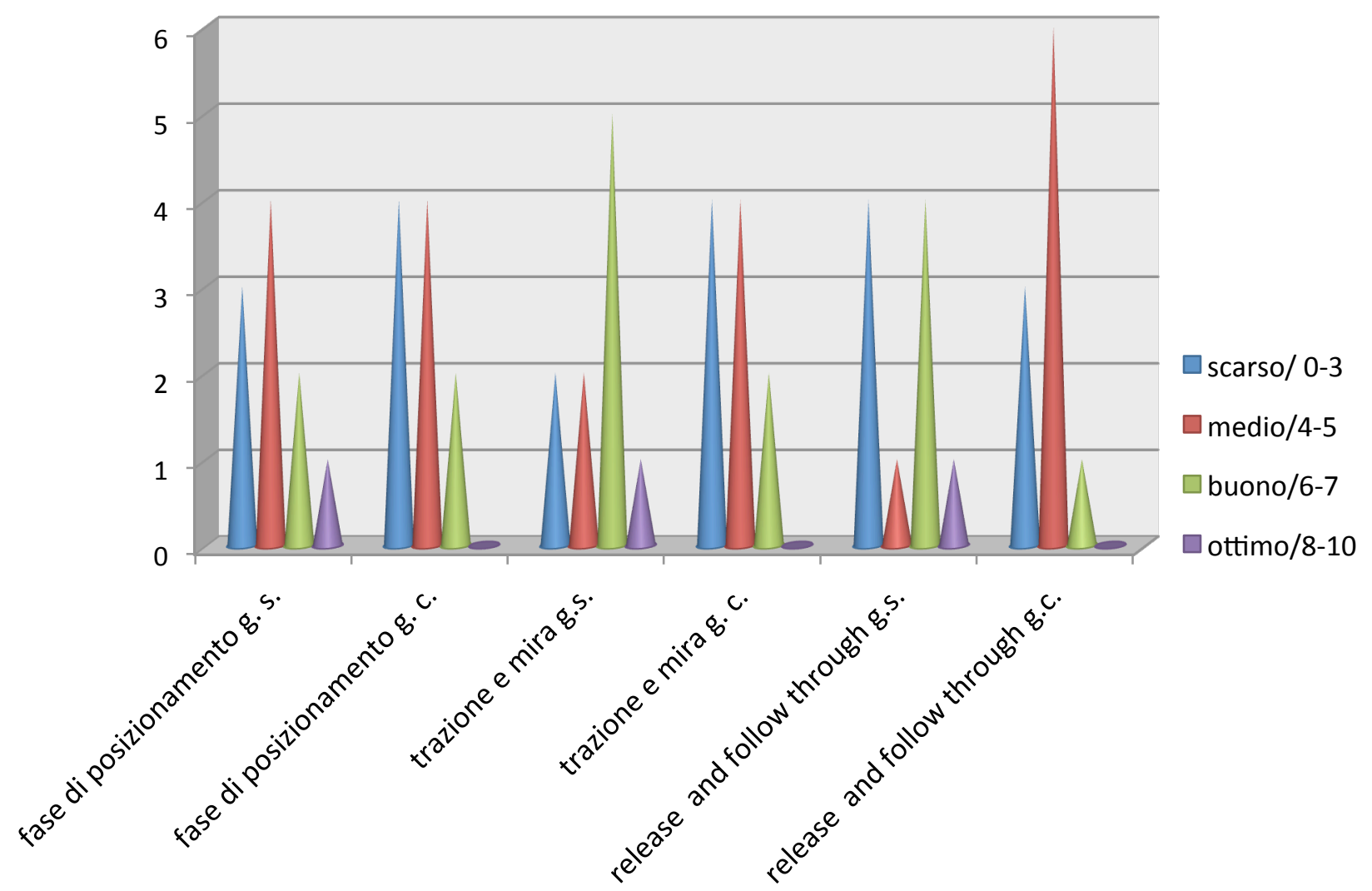

Figure 5. Final phase

\section{DISCUSSION}

In this study two basic aspects of the performance are examined: the motor execution and the motor imagine. Both share the same neuro-motor mechanism: the motor imagery. Concerning the woman artistic gymnastics, it can be useful during the training and the race. So providing the athletes and trainers of a means which uses the motor imagery as a possible application for the improvement of the performance. 


\section{CONCLUSIONS}

So in conclusion, the study aims to provide a standard training feasible on a large scale to train the cognitive and physical abilities of an athlete and provide a support tool in the race in order to improve performance, optimize time and to reduce the margin of error

\section{REFERENCES}

1. Galligan, F., et al. (2000). Acquiring Skill In: Galligan, F. et al., Advanced PE for Edexcel. 1st ed. Bath: Bath Press.

2. Knapp, B. (1967). Skill in Sport: The Attainment of Proficiency. London: Routledge \& Kegan Paul.

3. Raiola, G. (2012). La complessità dello studio in ambito sportivo. Lecce: Pensa.

4. Schmidt, R.A. \& Wrisberg, C. (2008). Motor Learning and Performance: A Situation-Based Learning Approach. USA: Human Kinetics.

5. Sanders, C.W., Sadoski, M., Bramson, R., Wiprud, R. \& Van Walsum, K. (2004). Comparing the effects of physical practice and mental imagery rehearsal in learning basic surgical skills by medical students. Am. J. Obstet Gyen, 191(5), pp.1811-4.

6. Tursi, D., Cerrotta, M., Napolitano, S. \& Raiola, G (2013). The video analysis as teaching tool in archery youth. Sport Sciences For Health, 9, pp. 56-57. 